\title{
DIFERENTES DENSIDADES DE CARGA EN TRUCHA ARCOIRIS MEDIANTE ANÁLISIS DE PARÁMETROS ECONÓMICOS Y BIOLÓGICOS
}

\author{
Rolando Chaves-Rojas ${ }^{1,}$ Ruth Vargas-Cordero ${ }^{2}$
}

\section{RESUMEN}

El principal objetivo de este experimento fue determinar la mejor densidad de carga en una compañía productora de truchas bajo la modalidad de raceways, mediante la determinación de parámetros biológicos y económicos. Se sembraron a cuatro densidades: $24,26,28$ y $30 \mathrm{~kg}$ de trucha/m³. Los peces se distribuyeron en jaulas flotantes ubicadas en dos estanques (E1 y E2) y organizadas en tres diferentes posiciones, de acuerdo a la entrada de agua (adelante, medio y atrás). Los estanques recibieron agua de segundo y tercer uso respectivamente. El periodo experimental fue de cuarenta y un días. Los parámetros calculados fueron: tasa de alimentación diaria, conversión alimenticia, tasa específica de crecimiento, coeficiente térmico de crecimiento, mortalidad, índice de conversión económico, margen bruto y relación margen/costo. Se analizó el efecto de la densidad en los diferentes parámetros, por medio de ANOVA, que contempló el estanque y la posición como factores. Los resultados reflejaron un crecimiento significativamente mayor en la densidad de $28 \mathrm{~kg} / \mathrm{m}^{3}$ en comparación a la de $30 \mathrm{~kg} / \mathrm{m}^{3}$, mientras que 24 y $26 \mathrm{~kg} / \mathrm{m}^{3}$ presentaron valores intermedios sin diferir significativamente entre sí, ni con las otras dos densidades. La densidad de $28 \mathrm{~kg} / \mathrm{m}^{3}$ mostró valores significativamente más beneficiosos en los parámetros económicos, que la de $24 \mathrm{~kg} / \mathrm{m}^{3}$; además, las densidades de 26 y $30 \mathrm{~kg} / \mathrm{m}^{3}$ se ubicaron en el medio sin mostrar diferencias significativas con las demás densidades. Por lo tanto, la mejor densidad de carga para truchas bajo las condiciones del experimento, es un rango entre 26 y $30 \mathrm{~kg} / \mathrm{m}^{3}$, pero se podrían realizar futuras investigaciones con densidades mayores. Asimismo, E1 presentó resultados significativamente mejores en todos los parámetros, en comparación a E2. Por otro lado, la posición atrás, la más alejada a la entrada de agua, obtuvo resultados más rentables que la posición adelante.

Palabras claves: densidad, truchas, peces, oxígeno, acuicultura

\footnotetext{
${ }^{1}$ Universidad de Costa Rica. Facultad de Ciencias Agroalimentarias. Estudiante de la Escuela de Zootecnia. Autor para correspondencia: roloch-2@hotmail.es

${ }^{2}$ Universidad de Costa Rica. Facultad de Ciencias Agroalimentarias. Estación Experimental Alfredo Volio Mata. Autor para correspondencia: ruth.vargas@ucr.ac.cr

Recibido: 3 de mayo 2016

Aceptado: 22 de agosto 2016
} 


\section{ABSTRACT}

The main objective of this research was to determine the best stoking density in a raceways trout commercial company by determination of biologic and economic parameters. Four treatments were used: 24, 26, 28 and $30 \mathrm{~kg}$ trout $/ \mathrm{m}^{3}$. Trout were organized in floating cages located in two different tanks (T1 and T2) and arranged in three different positions inside the tank, according to the water inlet (front, middle, and back). Tanks received water of second and third use, in that order. The experimental period was forty-one days. The economic and biological parameters calculated were: feed conversion ratio, specific growth rate, thermal-unit growth coefficient, mortality, economic conversion ratio, gross margin and margin/cost ratio. Stocking density effect on different parameters was analyzed by ANOVA. This statistical analysis took into account the tank and the position as factors. Results reflected a significantly better growth in $28 \mathrm{~kg} / \mathrm{m}^{3}$ density in comparison to $30 \mathrm{~kg} / \mathrm{m}^{3}$, while 24 and $26 \mathrm{~kg} / \mathrm{m}^{3}$ provided intermediate values without significant differences. Stocking density of $28 \mathrm{~kg} / \mathrm{m}^{3}$ showed more beneficial economic parameters than the $24 \mathrm{~kg} / \mathrm{m}^{3}$. In addition, stoking densities of 26 and $30 \mathrm{~kg} / \mathrm{m}^{3}$ were in the middle, without significant differences. Therefore, the best stoking density in this experiment for animals in cages is a range between 26 and $30 \mathrm{~kg} / \mathrm{m}^{3}$, but future research in the same conditions can focus on higher densities. Likewise, T1 reached significantly better results in most of the parameters than T2. On the other hand, the position back, the furthest from the water inlet, obtained more profitable results than position front.

Key words: density, trout, fish, oxygen, aquiculture. 


\section{INTRODUCCIÓN}

Las investigaciones sobre acuicultura son de suma importancia, dado el beneficio del consumo de peces y otras especies acuáticas en la alimentación humana (FAO 2004). En ese sentido, este tipo de producción es una excelente opción para nutrir a las futuras generaciones por dos razones; la creciente tasa de crecimiento de la población y el hecho de que la acuicultura genera mayores rendimientos por hectárea que las producciones terrestres (González 2002).

Paralelamente, al incentivarse esta actividad se generan efectos positivos para el ambiente al reducirse la sobreexplotación de los mares (González 2002). Por todo lo anterior, más empresas están invirtiendo en este tipo de negocio. Según Mejía y Mendoza (2013), dicho incremento se ha dado en diferentes sitios alrededor del mundo, incluyendo Costa Rica, y entre las especies que han sido soporte del mismo se encuentra la trucha arcoíris (Oncorhynchus mykiss).

Los principales factores que afectan la acuicultura son: calidad de agua, temperatura, oxígeno, genética, alimentación, sanidad y densidad de carga (Rengifo 2005). Con respecto a este último factor, se relaciona el peso de la biomasa y su volumen de crecimiento $\left(\mathrm{kg} / \mathrm{m}^{3}\right)$ (INCAGRO 2008). Cuando se incrementa esta proporción, algunos problemas pueden ocurrir: 1- los metabolitos acumulados afectan la calidad del agua, 2- la disponibilidad de comida puede ser limitada, 3- el apetito de los peces podría reducirse, 4el incremento del estrés fisiológico perjudica el funcionamiento del sistema digestivo (Granado 2000). Por lo tanto, es indispensable considerar el número de peces por metro cúbico con que se puede obtener el mayor beneficio económico.

Existen diferentes parámetros para determinar rendimientos económicos y biológicos, algunos de ellos se describen en los siguientes párrafos:

Tasa de alimentación diaria (TAD): porcentaje de consumo de alimento según el peso vivo del animal (Weatherley y Gill 1987).

Conversión alimenticia (CA o FCR por sus siglas en inglés Feed Convertion Ratio): kilogramos de alimento requeridos para incrementar un kilogramo de peso (Carrera 2010).

Tasa especifica de crecimiento (SGR por sus siglas en inglés Specific Growth Rate): refleja el porcentaje de crecimiento diario según el peso vivo del animal (Morales 2004). 
Coeficiente térmico de crecimiento (CTC): muestra una relación directa con el crecimiento; este parámetro es más preciso que el anterior (SGR), ya que considera el efecto de la temperatura del agua sobre el metabolismo animal. Esta medición es de suma importancia, debido a que la trucha es un animal poiquilotermo (Cho y Bureau, 1998).

Mortalidad: porcentaje de truchas muertas (Alvarado 1999).

Índice de conversión económico: costo económico requerido para producir un kilogramo de peso en el animal (Moñino, Tomás, et al., 2002).

Margen bruto: es la diferencia entre el ingreso económico por ventas del producto y el costo monetario en alimentación (Moñino et al., 2002).

Relación margen/costo: proporción entre el margen bruto y el costo económico invertido en el alimento (Moñino et al., 2002).

Por lo tanto, a efecto de determinar la mejor densidad de siembra en términos económicos y biológicos, el objetivo principal de esta investigación fue comparar tres densidades de siembra contra la que se utiliza en una empresa comercial; por lo que paralelamente se determinaron los parámetros fisicoquímicos del agua.

\section{MATERIALES Y MÉTODOS}

\section{Peces y Manejo}

Los animales fueron distribuidos en cuatro tratamientos utilizando 24 jaulas flotantes de un metro cúbico y a razón de 24, 26, 28 y $30 \mathrm{~kg}$ de trucha/m³, (D24, D26, D28 y D30 respectivamente), organizadas en 6 hapas por tratamiento y colocadas en 2 estanques (E1 y E2) para un total de 12 jaulas en cada uno. Las piletas no solo contenían las truchas en los encierros, sino también los peces de la empresa nadando libremente dentro de las mismas. Las jaulas se colocaron en tres diferentes posiciones dentro del tanque, de acuerdo a la entrada de agua (adelante, medio, atrás) (Figura 1, 2 y 3). La densidad utilizada por la empresa $\left(28 \mathrm{~kg} / \mathrm{m}^{3}\right)$ se consideró como tratamiento control, por lo que las restantes tres densidades fueron escogidas para determinar la densidad óptima, 
dependiendo de la decisión de aumentar o disminuir dicha cantidad para obtener la mejor ganancia. Los peces se mantuvieron en un periodo de aclimatación de 15 días.

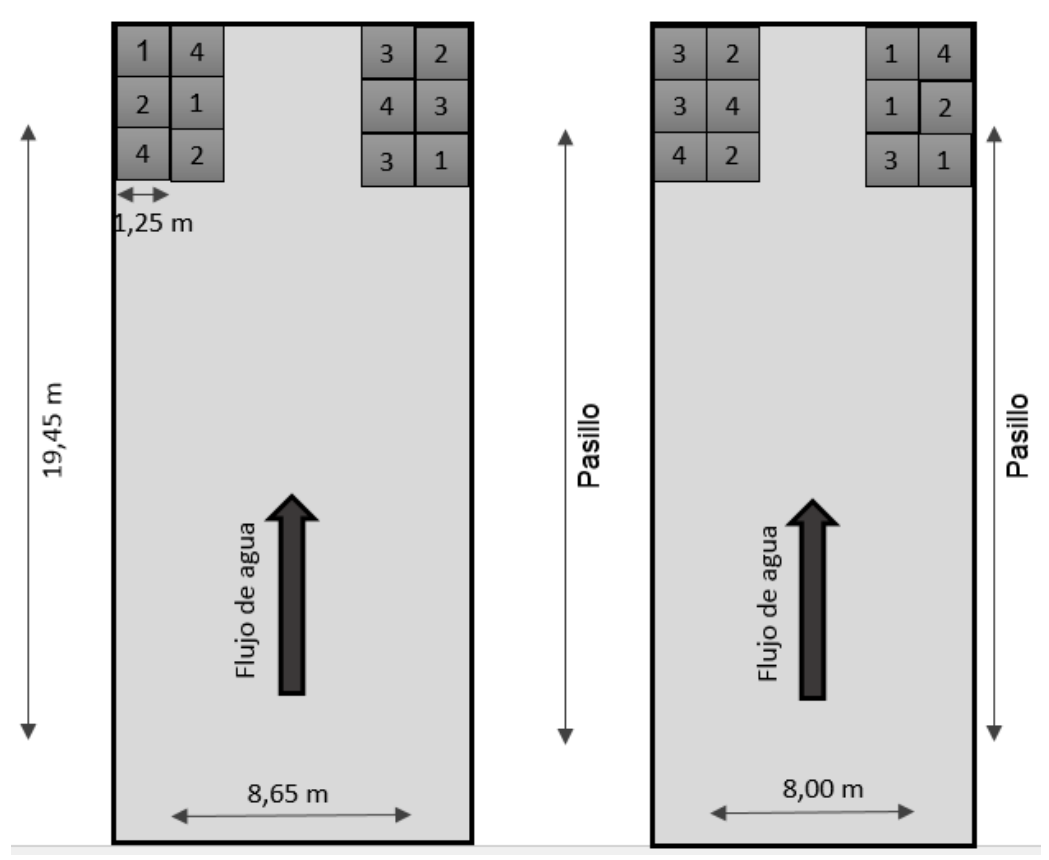

Figura 1. Distribución de jaulas en los estanques, según tratamientos: $1=24 \mathrm{~kg}$ trucha, $2=26 \mathrm{~kg}$ trucha, $3=28 \mathrm{~kg}$ trucha, $4=30 \mathrm{~kg}$ trucha.

La extensión del experimento fue de sesenta y ocho días; sin embargo, el periodo considerado para los cálculos fue de cuarenta y uno, sin considerar el periodo de aclimatación. Se excluyó de los cálculos veintidós días debido a problemas en la alimentación que comprometieron los resultados. El peso promedio de las truchas con que se contó para iniciar el estudio fue de $332 \pm 18$, obteniéndose al final del periodo experimental $397 \pm 14 \mathrm{~g}$ (peso a mercado).

El volumen de agua de los estanques fue de $193,5 \mathrm{~m}^{3}$ en E1 y de $205,4 \mathrm{~m}^{3}$ en E2. Estos recibieron agua de segundo y tercer uso respectivamente, a razón de aproximadamente $300 \mathrm{l} / \mathrm{s}$, por lo que la tasa de recambio de agua fue de 180\%/hora. En las siguientes figuras se observan fotografías de las instalaciones donde se realizó el ensayo. 
Nutrición Animal Tropical

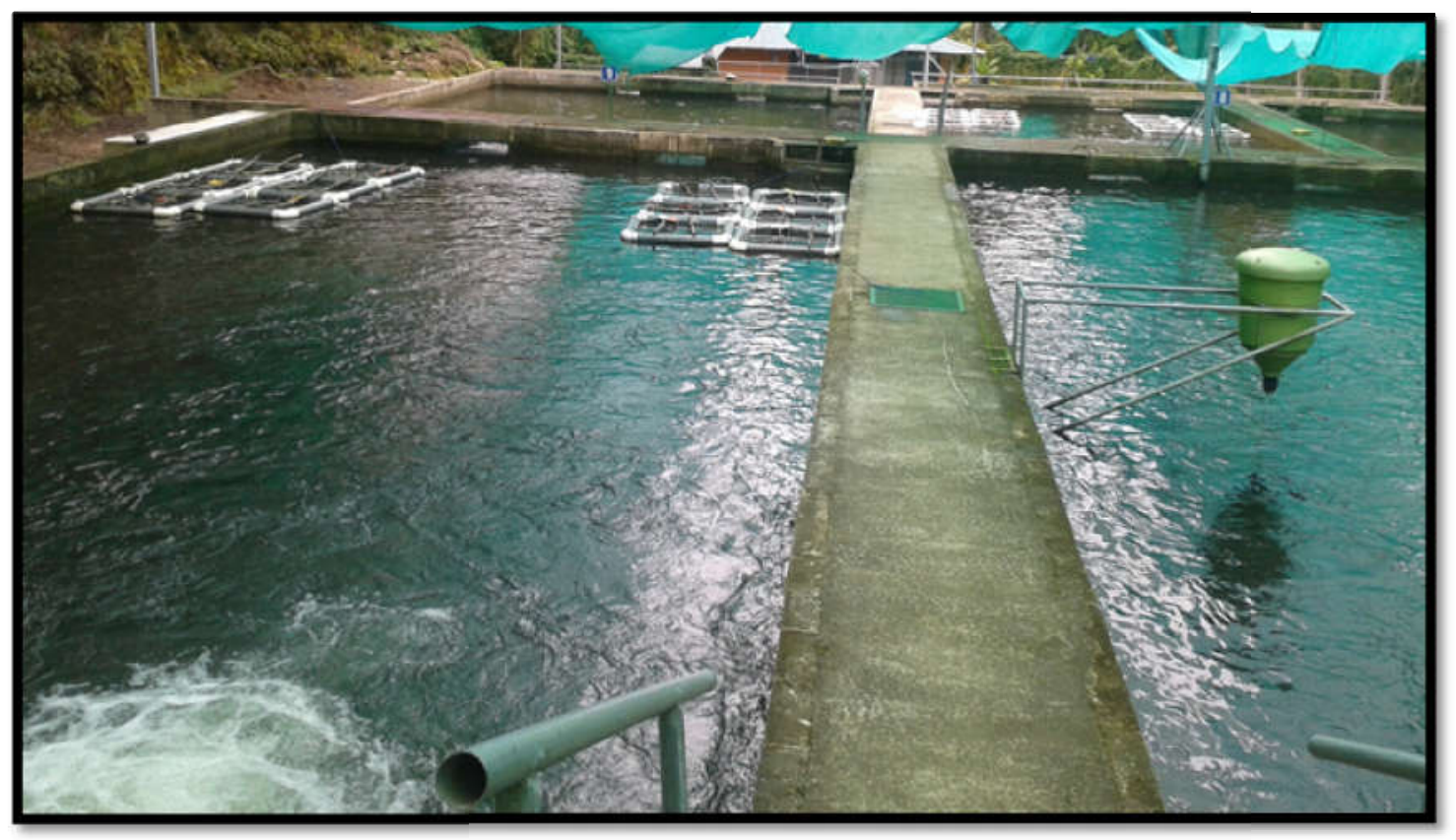

Figura 2. Ubicación de las jaulas dentro del proyecto truchícola.

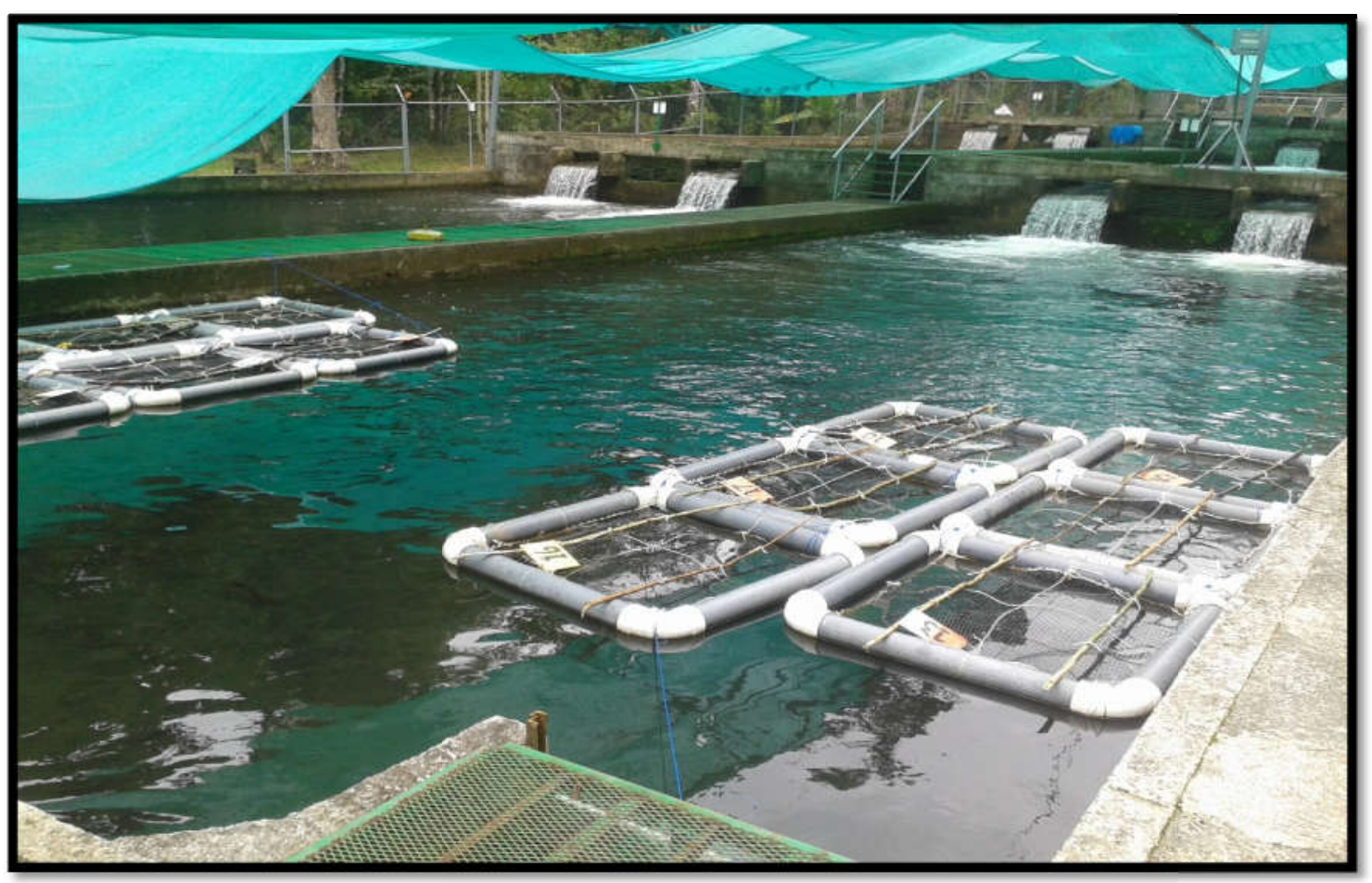

Figura 3. Jaulas del estanque 2. 


\section{Dieta y alimentación}

La composición de la dieta fue: $33,0 \%$ proteína cruda; $8,0 \%$ extracto etéreo; $6,0 \%$ fibra cruda; $10 \%$ cenizas; $10 \%$ humedad; 1 - 1,5\% calcio; $1,0 \%$ fósforo, $100 \mathrm{mg} / \mathrm{kg}$ vitamina C; $300 \mathrm{Ul} / \mathrm{kg}$ vitamina E, $4.514 \mathrm{kcal} / \mathrm{kg}$ energía digestible. Presentó además las siguientes características físicas: 7,0 mm el tamaño de partícula; 80,7 $\pm 12,52 \%$ flotabilidad y 2,11 \pm 0,94\% porcentaje de finos. Las truchas se alimentaron a saciedad, con una frecuencia de una, dos o tres veces al día (dependiendo de la turbidez del agua), cinco días por semana, esto es de lunes a viernes durante todo el periodo experimental.

Dado que durante el proceso de alimentación, las truchas en libertad dentro del tanque intentaban comer el alimento que se brindaba a los peces dentro de las jaulas, y por lo que podían robar los pellets a través de la red, se decidió agregar el alimento a las truchas dentro de las hapas, de manera más lenta, hasta que las mismas presentaran comportamiento de saciedad.

\section{Mediciones de temperatura y oxígeno en el agua}

Se realizaron mediciones de oxígeno tres veces al día (6:00 a.m., 12:00 m.d. y 6:00 p.m.) en cada jaula. Se registró la temperatura en el mismo horario, en la salida de agua de uno de los estanques. Se utilizó un oxigenómetro marca $\mathrm{YSI} 囚$, modelo 55-12FT, para la determinación de ambos valores.

\section{Parámetros}

Se calcularon diferentes parámetros económicos y biológicos, según las siguientes ecuaciones:

$$
\begin{gathered}
\text { Tasa de alimentación diaria }(T A D)=\frac{100 * \text { alimento consumido }}{\frac{\text { biomasa inicial + biomasa final }}{2}} / \text { días } \\
\text { Conversión alimenticia }(F C R)=\frac{\text { Alimento consumido }}{\text { ganancia de biomasa }} \\
\text { Tasa específica de crecimiento }(S G R)=\frac{((\ln (\text { peso final })-\ln (\text { peso inicial })}{\text { días }} * 100
\end{gathered}
$$




$$
\text { Coeficiente térmico de crecimiento }(\text { CTC })=\frac{(\text { Peso final ^ } 1 / 3-\text { peso inicial ^ } 1 / 3)}{\sum^{\circ} \mathrm{Ce}}
$$

Donde:

${ }^{\circ}$ Ce: Promedio diario de temperatura efectiva en ${ }^{\circ} \mathrm{C}$.

La temperatura efectiva es la resta entre el promedio de temperatura diaria y la temperatura mínima requerida por la especie para mantenimiento (Atwood, et al., 2003). La temperatura mínima requerida para el mantenimiento en truchas $3,8{ }^{\circ} \mathrm{C}$ (Elliott, 1982).

$$
\text { Mortalidad }=\frac{\text { Truchas muertas }}{\text { total de truchas iniciales }} * 100
$$

Índice de conversión económico $(I C E)=$ Precio del alimento $*$ FCR

Margen bruto $=$ ingreso por ventas - costo en alimentación

$$
\text { Relación margen costo }=\frac{\text { Margen bruto }}{\text { costo en alimentación }}
$$

El ajuste de la densidad en cada en cada jaula se realizó cada quince días, pesando todo el cardumen y para mantener la carga según cada tratamiento se extrajeron los peces necesarios que por incremento en peso hicieron que aquélla se sobrepasase al tratamiento. El término ganancia de biomasa en este experimento correspondió a la sumatoria de los kilogramos de peces extraídos por hapa en cada periodo.

Para el cálculo de los parámetros económicos, se consideró como producto la trucha entera, a un precio de $\$ 2,98$ el kilogramo; mientras que el costo del alimento, correspondió a $\$ 0,98 / \mathrm{kg}$.

\section{Análisis estadístico}

El efecto de la densidad en los diferentes parámetros, se analizó mediante un ANOVA. El modelo utilizado fue $Y_{i \mathrm{jkl}}=\mu+A_{i}+B_{j}+C_{k}+\varepsilon_{i j k l}$; donde $Y$ es la variable dependiente (cada parámetro), $\mu$ es el promedio general, A representa la densidad de carga, B es el 
estanque, $\mathrm{C}$ es la posición dentro del tanque y $\varepsilon$ corresponde al error experimental. Cuando se encontraron diferencias significativas, se utilizó la prueba de Tukey (1981), en caso de no detectarse mediante este análisis, se procedió a realizar la prueba de Duncan (1974). Para determinar normalidad en los datos, se aplicó la prueba de Shapiro-Wilk modificada (1997). Se utilizó la prueba de Kruskal Wallis (1952) cuando los datos no cumplieron con los supuestos del modelo. Se consideró un nivel de significancia del 95\% para indicar diferencias significativas. Todos los análisis se ejecutaron con el programa Infostat®, versión 2014.

\section{RESULTADOS Y DISCUSIÓN}

\section{Condiciones del agua}

En cuanto al oxígeno disuelto en el agua, no se encontraron diferencias significativas entre densidades ni entre posiciones, pero sí entre tanques (Cuadro 1).

Cuadro 1. Promedio de oxígeno disuelto $(\mathrm{mg} / \mathrm{l})$, según niveles estadísticos de cada factor.

\begin{tabular}{cccc}
\hline Factor & Nivel & Medias (mg/l) & Valor $\mathbf{p}$ \\
\hline \multirow{2}{*}{ Estanque } & E1 & $6,66^{\mathrm{b}}$ & $<0,0001$ \\
& E2 & $5,74^{\mathrm{a}}$ & \\
Posición & Adelante & 6,26 & \\
& Medio & 6,17 & \\
& Atrás & 6,17 & 0,7589 \\
Densidad & D24 & 6,22 & \\
& D26 & 6,16 & \\
& D28 & 6,24 & \\
& D30 & 6,17 & \\
\hline a,b Diferentes superíndices dentro del mismo factor, indican diferencias significativas (P<0,05).
\end{tabular}


Según el Cuadro 1 el oxígeno disuelto fue significativamente mayor en el E1 con respecto al E2. En la Figura 4 se ilustra el comportamiento del oxígeno en cada estanque, a lo largo del periodo.

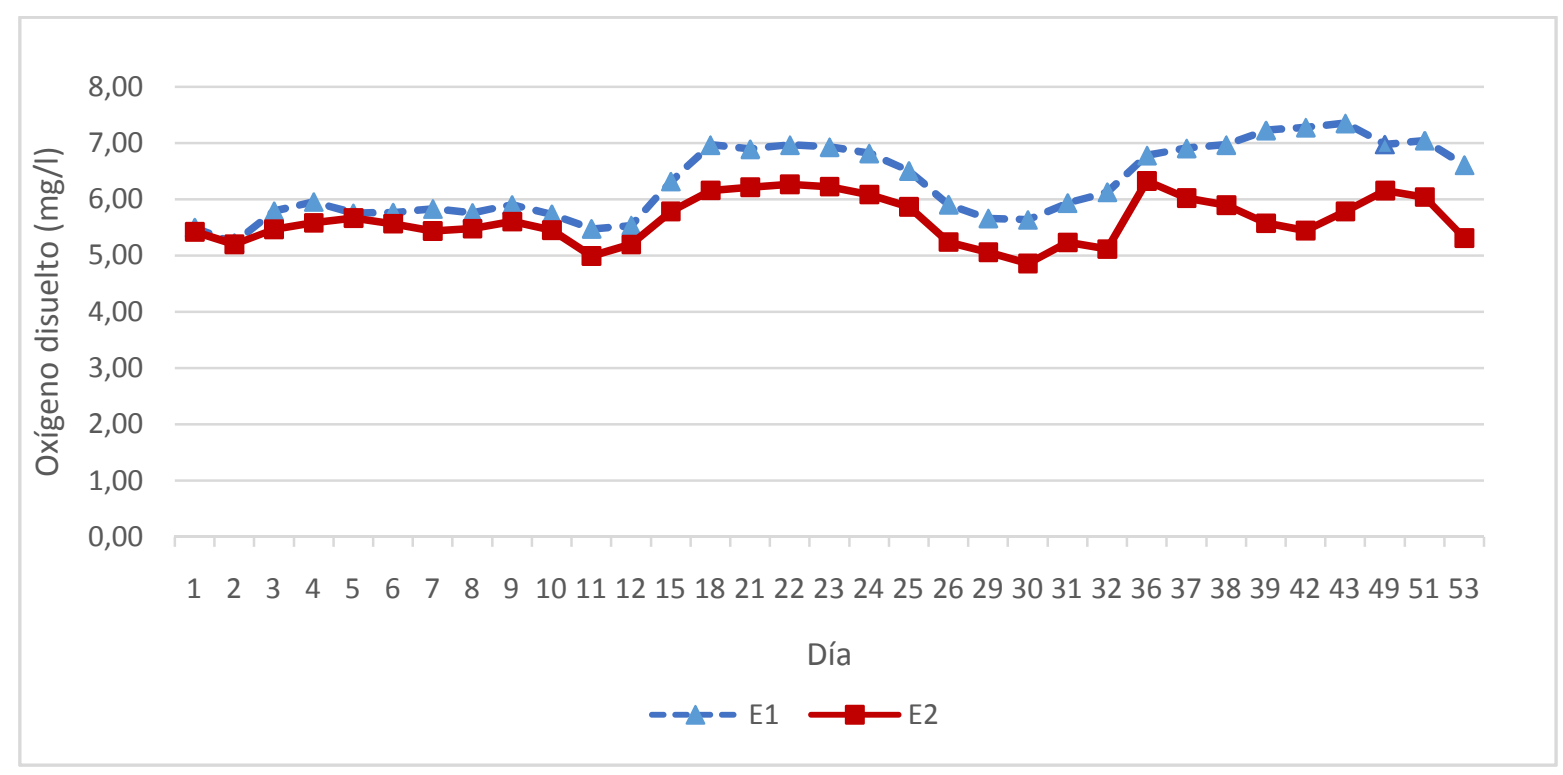

Figura 4. Resumen del promedio diario de concentración de oxígeno disuelto $(\mathrm{mg} / \mathrm{l})$ por estanque a lo a lo largo de cada semana.

En la Figura 4 se observa que la concentración promedio diaria de oxígeno fue superior en E1 en comparación con E2 durante todo el periodo. Esa diferencia entre estanques, se debe a la cantidad de usos del agua que suple a cada estanque, pues el E1 permaneció con agua de segundo uso, mientras que el E2 con agua de tercer uso.

Por otro lado, a lo largo del día, el oxígeno fue significativamente $(p<0,0001)$ mayor a las 6:00 comparado a los otros dos horarios de medición. En el Cuadro 2 de se detalla los resultados de este análisis. 
Cuadro 2. Oxígeno disuelto $(\mathrm{ml} / \mathrm{l})$ según horario de medición, por estanque

\begin{tabular}{cccc}
\hline Estanque & Hora & Promedios $(\mathbf{m g} / \mathbf{l})$ & Valor $\mathbf{p}$ \\
\hline E1 & $6: 00$ & $6,72^{\mathrm{b}}$ & $<0,0001$ \\
& $12: 00$ & $6,14^{\mathrm{a}}$ & \\
& $18: 00$ & $6,05^{\mathrm{a}}$ & \\
& & $<0,0001$ \\
E2 & $6: 00$ & $6,04^{\mathrm{b}}$ & \\
& $12: 00$ & $5,41^{\mathrm{a}}$ & \\
& $18: 00$ & $5,44^{\mathrm{a}}$ & \\
${ }^{\mathrm{a}, \mathrm{b}}$ Diferentes superíndices dentro del mismo factor, indican diferencias significativas $(\mathrm{P}<0,05)$
\end{tabular}

En el Cuadro 2 se evidencia claramente valores de oxígeno muy similares entre las mediciones correspondientes a las 12:00 y a las 18:00. Estos resultados se mantuvieron a lo largo del experimento.

La diferencia en oxígeno disuelto entre las 6:00 y los otros dos horarios, se debió a varias razones. En primera instancia, a los horarios de alimentación, pues estos fueron a partir de las 8:00., dos o tres veces al día; por lo que se promueve la reducción de oxígeno a partir de esta hora, ya que cuando los peces comen, incrementan su tasa de consumo de oxígeno para metabolizar el alimento (Alsop y Wood, 1997).

A las 6:00, el agua ha recuperado el oxígeno disuelto, pues la tasa de recambio en este experimento es bastante alta (aproximadamente 180\%/hora). Brito (2009) explica que altos recambios de agua inducen mejores niveles de oxígeno, por el hecho de la gran cantidad que ingresa a los estanques; es por esto que el impacto en el oxígeno disuelto se observó solamente cuando los peces aumentaban en gran medida su consumo de oxígeno.

Asimismo, el oxígeno disuelto presentó una relación inversa con la temperatura del agua, según el horario de medición. A las 6:00 (tiempo de medición en que la concentración de oxígeno fue mayor) se encontró la menor temperatura $\left(12,8 \pm 0,496{ }^{\circ} \mathrm{C}\right.$ en promedio). Mientras que a las $12: 00$ y a las $18: 00$, las concentraciones de oxígeno no variaron significativamente entre sí, pero fueron significantemente menores con respecto a las 6:00.; comparativamente la temperatura fue más elevada $\left(14,4 \pm 0,496\right.$ y $14,5 \pm 0,751{ }^{\circ} \mathrm{C}$ respectivamente). El comportamiento de la temperatura según el horario de medición, a lo largo del periodo, se detalla en la Figura 5. 


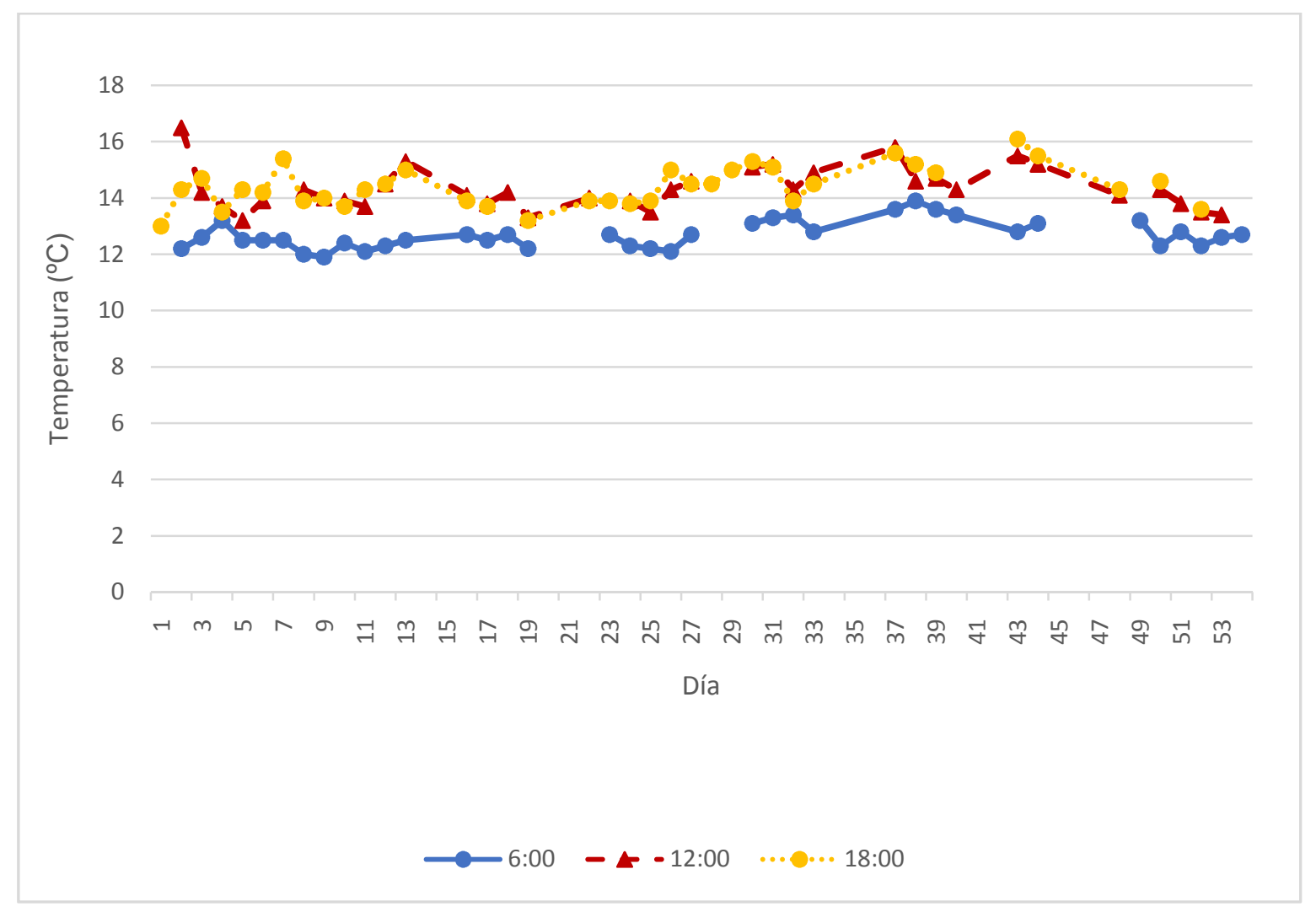

Figura 5. Temperatura del agua $\left({ }^{\circ} \mathrm{C}\right)$ según horario durante el periodo en consideración.

En este estudio, se evidencia lo mencionado por INCAGRO (2008), quien asegura que el oxígeno disuelto y la temperatura están inversamente relacionados. Esto se da debido a que, conforme la temperatura se reduce, la solubilidad del oxígeno se incrementa (Vega, et al. 1998). Asimismo, a medida que la temperatura se eleva, el metabolismo de los peces se acelera (Sanz y Zamora 2012), lo cual repercute en mayor consumo de oxígeno por parte de los mismos (Rincón 2008); consecuentemente, el oxígeno del agua decrece.

\section{Efecto de la densidad de carga}

Este factor provocó efectos significativos $(p<0,05)$ en los parámetros: SGR, CTC y margen bruto (Cuadro 3). 
Cuadro 3. Promedio de parámetros según densidad de carga

\begin{tabular}{|c|c|c|c|c|c|}
\hline \multirow[t]{2}{*}{ Parámetro } & \multicolumn{4}{|c|}{ Densidad de carga } & \multirow[t]{2}{*}{ Valor $p$} \\
\hline & $24 \mathrm{~kg} / \mathrm{m}^{3}$ & $26 \mathrm{~kg} / \mathrm{m}^{3}$ & $28 \mathrm{~kg} / \mathrm{m}^{3}$ & $30 \mathrm{~kg} / \mathrm{m}^{3}$ & \\
\hline TAD (\%) & 0,98 & 0,94 & 0,96 & 0,96 & 0,3942 \\
\hline CA & 2,51 & 2,32 & 2,16 & 2,34 & 0,108 \\
\hline SGR (\%) & $0,440^{\mathrm{ab}}$ & $0,431^{a}$ & $0,479^{b}$ & $0,425^{a}$ & 0,0469 \\
\hline СТС & $1,04 \mathrm{E}-04^{\mathrm{ab}}$ & $1,01 \mathrm{E}-04^{\mathrm{ab}}$ & 1,12E-04b & $9,92 \mathrm{E}-05^{\mathrm{a}}$ & 0,040 \\
\hline Mortalidad (\%) & 0,19 & 0,00 & 0,19 & 0,19 & 0,8333 \\
\hline $\operatorname{ICE}(\$)$ & 2,46 & 2,28 & 2,12 & 2,29 & 0,1082 \\
\hline Margen bruto $(\$)$ & $2,99^{\mathrm{a}}$ & $3,68^{\mathrm{ab}}$ & $5,10^{b}$ & $4,19^{\mathrm{ab}}$ & 0,015 \\
\hline $\begin{array}{c}\text { Relación } \\
\text { Margen/costo }\end{array}$ & 0,28 & 0,34 & 0,43 & 0,33 & 0,1077 \\
\hline \multicolumn{6}{|c|}{$\begin{array}{l}\text { No se encontraron diferencias significativas en las variables CA, ICE y relación } \\
\text { margen/costo utilizando un nivel de significancia del } 95 \% \text {. No obstante, al usar un valor } \\
\text { del } 90 \% \text {, en el nivel D28 se presentaron valores más cercanos a ser significativamente (el } \\
\text { valor p fue ligeramente mayor a } 0,10 \text { ) más beneficiosos que D } 24 \text { en los tres parámetros; } \\
\text { mientras que D26 y D30 presentaron valores intermedios sin diferir significativamente } \\
\text { entre sí, ni con las otras dos densidades. Por otro lado, la mortalidad no presentó } \\
\text { diferencias significativas entre las densidades, bajo ninguno de los dos niveles de } \\
\text { significancia utilizados. }\end{array}$} \\
\hline $\begin{array}{l}\text { Tal como se mues } \\
\text { significativamente er } \\
\text { apetito de los peces, } \\
\text { a esta contrariedad, } \\
\text { elevada y la baja tas }\end{array}$ & $\begin{array}{l}\text { en el Cua } \\
\text { densidades } \\
\text { desto a lo m€ } \\
\text { drían ser: la } \\
\text { alimentació }\end{array}$ & $\begin{array}{l}\text { ro } 4 \text {, la tas } \\
\text { lo cual quier } \\
\text { ncionado por } \\
\text { diferencia en } \\
\text { a la que fuer }\end{array}$ & $\begin{array}{l}\text { de alimen } \\
\text { decir que } \\
\text { anado (200 } \\
\text { densidade } \\
\text { alimentada }\end{array}$ & $\begin{array}{l}\text { ción diaria } \\
\text { te factor } \\
\text { Las posit } \\
\text { utilizadas } \\
\text { as truchas }\end{array}$ & $\begin{array}{l}\text { no difirió } \\
\text { afectó el } \\
\text { razones } \\
\text { es muy }\end{array}$ \\
\hline
\end{tabular}


Como se mencionó anteriormente, la TAD obtenida $(0,96 \%)$ fue muy baja en comparación a la indicada por Rengifo (2005), la cual es de 1,9\% para truchas del mismo peso y criados bajo la misma temperatura. Dicha baja TAD, fue producto del lento proceso de alimentación; debido a que esto promovió que los peces en las jaulas gastaran su energía tratando de capturar los pellets, por lo que estos parecían saciados, cuando en realidad no lo estaban. Para reforzar lo anterior, Toledo (1994), explica que los peces comen hasta cierto límite de tiempo después de iniciarse la descarga del alimento.

Dicho efecto de la alimentación, provocó una crecimiento muy lento, lo cual se evidencia con la baja tasa de crecimiento obtenida ( $S G R$ promedio $=0,444 \%$ ) en comparación a la alcanzada por Caballero, et al. (2002) (SGR $=1,81 \%$ ) con truchas de peso inicial de $247 \mathrm{~g}$ hasta alcanzar los $789 \mathrm{~g}$, a una temperatura de $11,9^{\circ} \mathrm{C}$, alimentados dos veces al día a saciedad. Asimismo, este argumento se refuerza con el valor conseguido de CTC, el cual, en promedio fue de 0,00011; siendo este mucho menor al determinado por Martínez et al. (2003), quienes obtuvieron un CTC de 0,00165, en truchas de 0,4 g como peso inicial y $250 \mathrm{~g}$ como peso final, a $10,5^{\circ} \mathrm{C}$ y $5,5 \mathrm{mg} / \mathrm{l}$ de oxígeno. Aunque si bien es cierto, en dicho experimento la edad de los animales fue menor; siendo que, según Aguilar (2010), estos animales presentan un crecimiento más acelerado en las fases iniciales, por lo que comparativamente van a presentar mayores CTC; se considera sin embargo, que la diferencia no debería ser de tal magnitud $(0,00165$ vs 0,00011$)$.

Ligado a lo anterior, del trabajo de Venegas, et al. (2008); se obtiene un CTC de 0,00138, con truchas de mayor edad, de un peso promedio inicial de $704 \mathrm{~g}$ y un peso final de 1049 g. Bajo una temperatura media de $18,3^{\circ} \mathrm{C}$ y un oxígeno disuelto de $7,11 \mathrm{mg} / \mathrm{l}$. En dicho caso, pese a que los animales presentan una edad superior a los de este experimento, el CTC obtenido fue mayor; aunque los peces tuvieron las ventajas de mantenerse bajo condiciones más favorables de oxígeno y temperatura; es otro claro ejemplo en el que, comparativamente se refleja en la literatura, valores muy superiores de CTC con respecto al del presente estudio.

A pesar de lo explicado en los párrafos anteriores, si se observó un efecto de la densidad en el crecimiento de estos animales. La tasa de crecimiento en D28 (SGR $=0,479 \%$ ) fue significativamente $(p=0,0469)$ mayor a D26 y $D 30 \quad(S G R=0,431$ y $0,425 \%$ respectivamente); mientras que D24 ( $S G R=0,440 \%$ ) se ubicó en la segunda posición sin diferir significativamente de las otras tres densidades. Además el CTC en D28 $(1,12 \mathrm{E}-04)$ 
fue significativamente $(p=0,0406)$ mayor al de D30 (9,92E-05), mostrando D24 y D26 valores intermedios (1,04E-04 y 1,01E-04 respectivamente) sin diferir significativamente entre sí, ni con los otros dos niveles.

Por lo tanto, en este estudio se evidencia que una de las densidades intermedias (D28) presentó el crecimiento más rápido. Resultados similares encontró Grayeb del Älamo (2001) con Paralabrax maculatofasciatus criadas en jaulas flotantes; en ese estudio se determinó un crecimiento más acelerado en la densidad intermedia $\left(70\right.$ peces/ $\left.\mathrm{m}^{3}\right)$ en comparación con las otras (35 peces $/ \mathrm{m}^{3}$ y 105 peces $/ \mathrm{m}^{3}$ ). Además, Chua y Teng (1979) también encontraron algo semejante con Epinephelus salmoides bajo el mismo sistema productivo; en este caso, la densidad más óptima en los diferentes parámetros económicos fue la de 60 peces $/ \mathrm{m}^{3}$, la cual fue contrastada contra densidades de 30,90 y 120 individuos $/ \mathrm{m}^{3}$.

Estos resultados se pueden justificar aclarando que los peces son organismos sociales; por lo tanto, estos crecen con mayor velocidad cuando son criados con otros especímenes (porque deben competir para alimentarse), siempre y cuando su número sea adecuado. Entonces, no necesariamente el crecimiento va a ser mayor en densidades menores (Weatherley y Gill, 1987).

Como otra consecuencia del proceso de alimentación, se obtuvieron conversiones alimenticias muy elevadas $(C A=2,3)$, en comparación al valor normal de dicho parámetro para este tipo de peces (salmónidos), cuyo dato según Luchini y Panné (2008) es de 1,4 a 1. Otra posible causa a estos altos valores de CA, radica en que los peces en jaulas flotantes no pueden consumir todo el alimento, incluso si los pellets son ofrecidos cuidadosamente; ya que algunos de los gránulos salen de la superficie de la jaula antes de ser capturados (Grayb del Alamo, 2001).

En el parámetro margen bruto, se observó un valor significativamente $(p<0,05)$ más favorable para D28, en comparación a D24; simultáneamente D26 y D30 presentaron valores intermedios sin diferir significativamente entre sí, ni con las otras dos densidades. Mientras que para las variables: conversión alimenticia, índice de conversión económico y relación margen/costo; se evidenciaron resultados similares, en los cuales se obtuvo un beneficio cercano a ser significativo en D24 versus D28 y el mismo comportamiento en D26 y D30 al observado en el margen bruto. 
Por lo tanto, se puede destacar que las densidades más óptimas para truchas criadas bajo las condiciones descritas, son: 26,28 y $30 \mathrm{~kg} / \mathrm{m}^{3}$ (un rango de 26 a $30 \mathrm{~kg} / \mathrm{m}^{3}$ ). Esto coincide con Guerrero (2006), quien recomendó una densidad entre 25 y $30 \mathrm{~kg} / \mathrm{m}^{3}$ para truchas en jaulas flotantes en la fase de engorde. Asimismo, los resultados se encuentran dentro del rango sugerido por Perdomo, et al. (2011), el cual es de 25 a $40 \mathrm{~kg} / \mathrm{m}^{3}$ para animales de esta misma especie, en cualquier fase productiva.

\section{Efecto del estanque}

Entre los estanques se encontraron diferencias significativas $(p<0,05)$ en todos los parámetros, excepto en la mortalidad y en la TAD (Cuadro 4).

Cuadro 4. Promedios de parámetros según el estanque.

\begin{tabular}{cccc}
\hline Parámetro & E1 & Estanque & Valor $\mathbf{p}$ \\
\cline { 2 - 3 } TAD (\%) & 0,97 & 0,95 & 0,3659 \\
CA & $1,99^{\mathrm{a}}$ & $2,68^{\mathrm{b}}$ & $<0,0001$ \\
SGR (\%) & $0,506^{\mathrm{b}}$ & $0,382^{\mathrm{a}}$ & $<0,0001$ \\
CTC & $1,18 \mathrm{E}-04^{\mathrm{b}}$ & $9,06 \mathrm{E}-05^{\mathrm{a}}$ & $<0,0001$ \\
Mortalidad (\%) & 0,10 & 0,19 & 0,5984 \\
ICE $(\$)$ & $1,95^{\mathrm{a}}$ & $2,63^{\mathrm{b}}$ & $<0,0001$ \\
Margen bruto $(\$)$ & $6,24^{\mathrm{b}}$ & $1,74^{\mathrm{a}}$ & 0,0159 \\
Relación & $0,53^{\mathrm{b}}$ & $0,15^{\mathrm{a}}$ & $<0,0001$ \\
\hline Margen/costo & & & \\
\hline a,b & Diferentes superíndices dentro del mismo factor, indican diferencias significativas $(\mathrm{P}<0,05)$.
\end{tabular}

En el Cuadro 4 se puede observar que el E1 mostró significativamente $(p<0,05)$ mejores resultados, en crecimiento y parámetros económicos, que el E2. Estos resultados están relacionados con la concentración de oxígeno, la cual fue significativamente $(p=0,0001)$ mayor en el primero en comparación al segundo. 
Por lo tanto, se puede asociar el componente oxígeno, como el principal determinante de los resultados obtenidos. Esto se explica al comprender de manera general la fisiología respiratoria de los peces. Las branquias, responsables del intercambio gaseoso entre la sangre y el agua (Martínez-Pereda 1996); sufren complicaciones en su labor conforme el oxígeno del agua disminuye (Guerrero 2006). Consecuentemente, decrecen los niveles de consumo de oxígeno por parte del organismo, lo que provoca que la producción de ATP (energía) en las mitocondrias se torne ineficiente; lo cual afecta los procesos metabólicos como el crecimiento (Luna-Ortiz, et al. 2013); reflejado en conversiones alimenticias elevadas, que inducen bajos índices económicos (Brito 2009). Según Carrera (2010), el rango óptimo de oxígeno disuelto en truchas, es de 7 a 9 mg/l.

Una manera de contrarrestar la disminución de oxígeno a lo largo del día, o la baja en su concentración debido a la reutilización del agua; es por medio de aireación. Esta puede ser natural (caídas de agua, escaleras, chorros, cascadas y sistemas de abanico) o mecánica (motobombas, difusores, aireadores de paleta, aireadores de inyección de oxígeno y generadores de oxígeno líquido) (Brito 2009). De hecho, según el mismo autor, con una buena aireación se puede alcanzar un incremento en la densidad de siembra de hasta $30 \%$. 


\section{Efecto de la posición}

El efecto de la posición de la jaula de acuerdo a la entrada de agua, no fue significativo $(p>0,05)$ para ninguna de las variables (Cuadro 5$)$.

Cuadro 5. Promedio de parámetros según posición de la jaula.

\begin{tabular}{ccccc}
\hline \multirow{2}{*}{ Parámetro } & \multicolumn{3}{c}{ Posición según la entrada de agua } & \multirow{2}{*}{ Valor $\mathbf{p}$} \\
\cline { 2 - 3 } & Adelante & Medio & Atrás & \\
TAD (\%) & 0,97 & 0,94 & 0,97 & 0,3333 \\
CA & 2,40 & 2,38 & 2,22 & 0,2569 \\
SGR (\%) & 0,445 & 0,445 & 0,442 & 0,9812 \\
CTC & $1,05 \mathrm{E}-04$ & $1,05 \mathrm{E}-04$ & $1,04 \mathrm{E}-04$ & 0,9622 \\
Mortalidad (\%) & 0,14 & 0,14 & 0,14 & $>0,9999$ \\
ICE $(\$)$ & 2,35 & 2,33 & 2,18 & 0,2569 \\
Margen bruto $(\$)$ & 3,50 & 3,74 & 4,73 & 0,0635 \\
$\begin{array}{c}\text { Relación } \\
\text { Margen/costo }\end{array}$ & 0,30 & 0,33 & 0,40 & 0,1360 \\
\hline
\end{tabular}

Aunque en el Cuadro 5 no se observan diferencias significativas en ninguna de las variables $(p>0,05)$, con un nivel de significancia del $90 \%$ se detectó en el parámetro margen bruto un valor significativamente mayor en la posición atrás con respecto a la posición adelante, mientras que la posición medio mostró un número intermedio que no difirió significativamente de las otras dos posiciones.

Una posible razón que justifica los resultados en dicho parámetro es que a medida que las jaulas se posicionaron más cerca a la entrada de agua, la corriente fue más fuerte; por lo tanto, las truchas gastaron más energía en su locomoción, la cual, según Castelló (1993), es una de las actividades más costosas para el metabolismo de los peces. Consecuentemente quedó disponible menor energía para el crecimiento, lo que repercute en una menor ganancia económica. 


\section{CONSIDERACIONES FINALES}

En este estudio se concluyó que el incremento de peso depende del número ideal de peces por metro cúbico en el que se dé la adecuada competencia por el alimento y a su vez no se afecte de manera negativa la calidad del agua, la accesibilidad del alimento, el apetito de los peces y la capacidad digestiva de los mismos. Con base en ello, se evidenció que la densidad ideal para truchas bajo las condiciones del ensayo, es un rango de 26 a $30 \mathrm{~kg} / \mathrm{m}^{3}$; incluso se podrían realizar futuras investigaciones probando densidades mayores.

Además, se comprobó que conforme se mantengan las truchas bajo una concentración de oxígeno más cercana a su rango óptimo, mayor ventaja se obtiene en los parámetros biológicos y económicos. Para contrarrestar concentraciones bajas de $\mathrm{O}_{2}$, se puede optar por sistemas de aireación de agua; esto permite inclusive manejar densidades mayores.

Por otro lado, en este ensayo también se observó, cómo la fuerza de la corriente de agua puede repercutir en los resultados económicos, demostrado en este estudio con el parámetro margen bruto; en el cual el beneficio fue muy cercano a ser significativamente mayor, a menor corriente de agua. Razón que se puede atribuir al menor costo energético por parte de los peces.

Como recomendación para futuros experimentos en jaulas flotantes, se debe delimitar, con una red el perímetro en el que se ubican las jaulas; para así evitar la intervención de los peces libres dentro del estanque, durante el proceso de alimentación. 


\section{LITERATURA CITADA}

Aguilar, F. 2010. Modelos matemáticos no lineales como herramienta para evaluar el crecimiento de tilapia roja (Oreochromis spp.) y tilapia nilótica (Oreochromis niloticus var. Chitralada) alimentadas con dietas peletizadas o extruidas. Tesis de Magíster en Producción Animal, Universidad Nacional de Colombia. Bogotá, Colombia. 135 pp.

Alsop, D. y C. Wood. 1997. El efecto de la interacción entre la alimentación y el ejercicio, en el consumo de oxígeno, comportamiento en natación y uso de proteína, en juveniles de truchas arcoíris (Oncorhynchus mykiss). (En inglés) The Journal of Experimental Biology. 200:2337-2346.

Alvarado, H. 1999. Crecimiento de la trucha arcoíris cultivada en diferentes tipos de estanques y densidades. Veterinaria Tropical. 24(2):121-129.

Atwood, H., J. Tomasso,K. Webb y D. Gatling. 2003. Tolerancia de tilapia Nilótica (Oreochromis niloticus) a bajas temperaturas: efectos de los factores ambiente y dieta. (En inglés) Aquaculture Research 34: 241-251

Brito, F. 2009. Efecto de la reutilización del agua en la crianza y producción de tilapia roja. Tesis de Grado. Universidad del Azuay. Ecuador. 63 p.

Caballero, M., A. Obach, G. Rosenlund, D. Montero, M. Gisvold y M. izquierdo. 2002. Impacto de diferentes fuentes de lípidos, en crecimiento, digestibilidad de lípidos, composición de ácidos grasos del tejido e histología de trucha arcoíris Oncorhynchus mykiss. Aquaculture (En inglés). 214(1-4):253-271.

Carrera,S. 2010. Evaluación de dos densidades de siembra y dos fuentes de proteína (lombrices y vísceras de pollo) en la producción de trucha "Arco Iris" (Oncorhynchus mykiss). Tesis de Grado. Universidad Nacional de Loja. Ecuador. $109 \mathrm{p}$.

Castelló, F. 1993. Acuicultura marina: Fundamentos biológicos y tecnología de la producción. Universidad de Barcelona. Barcelona, España. 747 p.

Cho, C y D. Bureau. 1998. Desarrollo de modelos bioenergéticos y el programa FishPrFEQ para estimar producción, ración de alimentación y producción de residuos en acuicultura. (En inglés) Aquatic Living Resource. 11(4): 199-210. 
Chua, T y S. Teng. 1979. Crecimiento relativo y producción del mero estuario Epinephelus salmoides bajo diferentes densidades de carga, en jaulas flotantes de red. (En inglés)Marine Biology. 54(4):363-372.

Di Rienzo, J.A, F. Casanoves, M. Balzarini, L. González, M. Tablada y C. Robledo. Infostat versión 2014. Grupo InfoStat, FCA, Universidad Nacional de Córdoba, Argentina. URL http://www.infostat.com.ar.

Duncan, A.1974. Control de calidad y estadísticas industriales. (En inglés) 4th Ed., Irwin, Homewoods, III.

Elliott, J. 1982. El efecto de la temperatura y talla, en el crecimiento y la energética de salmónidos en cautiverio. (En inglés) Comp. Biochem. Physiol. 73B(1):81-91.

FAO. 2004. El estado mundial de la pesca y la acuicultura. (En línea). Consultado el 10 de abril del 2013. Disponible en: http://www.fao.org/docrep/007/y5600s/y5600s00.htm\#TopOfPage. 172972.

González, F. 2002. La acuicultura y el desarrollo económico. Boletín Instituto español de oceanografía.18 (1-4). 265-274 p.

Granado, A. 2000. Efecto de la densidad de cultivo sobre el crecimiento del morocoto, Piaractus brachypomus, Cuvier 1818, (Pisces: Characiformes), confinado en jaulas flotantes. Saber. 12(2):3.7.

Grayeb del Álamo, T. 2001. Efecto de la densidad en el crecimiento de la cabrilla arenera Paralabrax maculatofasciatus (Percoidei: Serranidae) cultivada en jaulas flotantes. Tesis de Maestría. Instituto Politécnico Nacional. La Paz, México. 119 p.

Guerrero, J. 2006. La nutrición y alimentación eficiente de los peces. (En línea). Consultado el 05-02-15. Disponible en: ftp://ftp.fao.org/fi/CDrom/FAO training/FAO training/general/x6709s/x6709s10.htm Incagro e Inversiones Santa Inés SAC. 2008. Manual para la producción de truchas en jaulas flotantes. (En línea). Consultado el 14-01-15. Disponible en: http://www.bvcooperacion.pe/biblioteca/bitstream/123456789/946/1/BVCI0000802. pdf.

Kruskal, W y W. Wallis. 1952. El uso de filas en el análisis de varianza de un sólo criterio.(En inglés) Journal of the American Statistical Association, 47: 583-621.

Luchini, L y S. Panné. 2008. Perspectivas en acuicultura; Nivel mundial, regional y local. Secretaría de agricultura, ganadería, pesca y alimentos. Argentina. (En línea). Consultado el 7-nov-2015. Disponible en: 


\section{http://produccionbovina.com.ar/produccion peces/piscicultura/113-}

perspectivas.pdf.

Luna-Ortiz, P., M. El-Hafidi yM. Martínez-Rosas. 2013. La función mitocondrial y la cardioprotección. Revista Mexicana de Anestesiología. 36(4):294-305.

Mahibbur, R yz. Govindarajulu. 1997. Una modificación del test de normalidad de Shapiro y Wilks. (En inglés)Journal of Applied Statistics, 24(2): 219-235.

Martínez-Pereda,J. 1996. La perfusión de branquias como modelo de valoración de la calidad de agua y su relación con la producción intensiva de truchas. Tesis de doctorado. Universidad Complutense de Madrid. Madrid, España. 142 p.

Martínez, S., A. Tomás, L. Pérez y M. Jover. 2003. Alternativas de diseño de una granja de truchas: volumen de producción y número de lotes anuales con dos perfiles de temperaturas. Revista AquaTIC. 19:35-40.

Mejía, H yV. Mendoza. 2013. Estudio de factibilidad de la creación de una estación experimental de producción y comercialización de truchas para el colegio provincia de Los Lagos en la parroquia San Francisco de Sigfipamba cantón PimampiroImbabura periodo 2012-2015. Tesis de Grado. Universidad Central de Ecuador. Quito, Ecuador. $154 \mathrm{p}$.

Miller, R. 1981.Inferencia estadística simultánea.(En inglés)2nd Ed. Springer-Verlag, Heidelberg and Berlin.

Moñino, A., A. Tomás, M. Fernández, R. lázaro, L. Pérez, F. Espinós, A. Tiana y M. Jover. 2002. Estudio del crecimiento, del aprovechamiento nutritivo y de la productividad económica de la dorada Sparus auratus L., 1758 alimentada con piensos comerciales de diferente contenido en proteína y lípidos. Boletín Instituto Español de Oceanografía. 18(1-4):275-280.

Morales, G. 2004. Crecimiento y eficiencia alimentaria de trucha arco iris (Oncorhynchus mykiss) en jaulas bajo diferentes regímenes de alimentación. Trabajo de grado. Universidad de Buenos Aires. Argentina. $51 \mathrm{p}$.

Perdomo, D., M. González, K. Castellanos, J. Gechele y F. Perea. 2011. Caracterización e índices productivos de granjas truchícolas en el Estado Trujillo Venezuela. Agricultura Andina. 19:17-29.

Rengifo, W. 2005. Montaje de una unidad productiva para la producción y comercialización de trucha ahumada, en la vereda La Palma, resguardo de 
Paniquita, municipio de Totoro, departamento del Cauca. Tesis de grado. Universidad Nacional Abierta y a Distancia. Popayán, Colombia. 58 p.

Rincón, R. 2008. Determinación de las relaciones existentes entre la temperatura ambiental, la masa visceral y corporal, la longitud corporal, el área muscular y la frecuencia respiratoria de Carassius auratus. Tesis de Grado. Universidad Militar Nueva Granada. Colombia. $51 \mathrm{p}$.

Sanz, F y S. Zamora. 2012. La nutrición y alimentación en piscicultura. Madrid Fundación Observatorio Español Acuicultura 2009. España. 804 p.

Toledo, M. 1994. Calidad física y química de los alimentos para peces y su importancia en el cultivo de peces. FAO. México. (En línea). Consultado el 06-10-2015. Disponible en: http://www.fao.org/docrep/field/003/ab482s/AB482S17.htm.

Vega, M., R. Pardo, E. Barrado y L. Debán. 1998. Evaluación de los efectos estacionales y de contaminación, en la calidad de agua de río, por análisis exploratorio de datos. (En inglés) Water Research. 32(12):3581-3592.

Venegas, P., C. Sobenes, C. Ortiz. 2008. Efecto de Tiempos de Retención Hidráulica de Agua, en un Sistema de Cultivo Experimental de un Paso para Trucha Arcoiris (Oncorhynchus mykiss). Información Tecnológica. 19 (6):71-82.

Weatherley, A., H. Gill. 1987. La biología del crecimiento del pez. (En inglés) Academic Press. Inglaterra.443 p. 